\title{
Small-time asymptotics for an uncorrelated local-stochastic volatility model
}

\author{
Martin Forde* Antoine Jacquier ${ }^{\dagger}$
}

20th April 2011

\begin{abstract}
We add some rigour to the work of Henry-Labordère[20], Lewis[25] and Paulot[28] on the small-time behaviour of a local-stochastic volatility model with zero correlation at leading order. We do this using the Freidlin-Wentzell (FW) theory of large deviations for SDEs, and then converting to a differential geometry problem of computing the shortest geodesic from a point to a vertical line on a Riemmanian manifold, whose metric is induced by the inverse of the diffusion coefficient. The solution to this variable endpoint problem is obtained using a transversality condition, where the geodesic is perpendicular to the vertical line under the aforementioned metric. We then establish the corresponding small-time asymptotic behaviour for call options using Hölder's inequality, and the implied volatility (using a general result in [30]). We also derive a series expansion for the implied volatility in the small-maturity limit, in powers of the log-moneyness, and we show how to calibrate such a model to the observed implied volatility smile in the small-maturity limit.
\end{abstract}

\section{Introduction}

Large deviations theory provides a natural framework for approximating the exponentially small probabilities associated with the behaviour of a diffusion process over a small time interval. In the context of Mathematical Finance, large deviations theory arises in the computation of small-maturity, out-of-the-money call or put option prices, or the probability of reaching a barrier or default level in a small time period. In recent years there has been an explosion of literature on small-time asymptotics for stochastic volatility models (see [1],[2],[12],[13],[14],[17],[18],[20],[21],[28],[29],[31]). All these articles characterize the behaviour of the Black-Scholes implied volatility for European options in the small maturity limit, and they are essentially applications and/or higher order corrections to the seminal work of Varadhan[32],[33] and the Freidlin-Wentzell theory of large deviations for stochastic differential equations[16], the main point being that the small-time behaviour of a diffusion process can be characterized in terms of an Energy/distance function on a Riemmanian manifold, whose metric is induced from the inverse of the diffusion coefficient. In Theorems 1.2 and 2.3 in Berestycki et al.[2], the authors show that for a stochastic volatility model with coefficients satisfying certain growth conditions, the implied volatility can be calculated in terms of a distance function which is the unique viscosity solution to a non-linear eikonal first order Hamilton-Jacobi PDE.

Henry-Labordère[20] formally computed a small-time expansion for the effective local volatility and the implied volatility for a general stochastic volatility model, using the expansion for the heat kernel on a Riemmanian manifold due to Minakshisundaram\&Pleijel[27], and then applying Laplace's method in the small-time limit when integrating over the range of the instantaneous volatility variable. Paulot[28] derived a small-time expansion for call options as well, by applying Laplace's method to integrate the heat kernel over the range of the volatility variable, then using the Tanaka-Meyer formula and some well known asymptotic expansions for the standard Normal distribution function. However, both these authors do not justify the integration over the infinite range of the volatility variable with appropriate tail estimates, and [20] does not prove existence and uniqueness for the associated differential geometry problem of computing the shortest geodesic to a line.

Forde\&Jacquier[13] made rigorous the work of Lewis[25] and Durrleman[9] on the small-time asymptotic behaviour of the implied volatility under the Heston stochastic volatility model. They applied the Gärtner-Ellis theorem from large deviations theory to the exponential affine closed-form expression for the moment generating function of the log

\footnotetext{
${ }^{*}$ Department of Mathematical Sciences, Dublin City University (Martin.Forde@dcu.ie), work supported by SFI grant for the Edgeworth Centre for Financial Mathematics.

${ }^{\dagger}$ Institut für Mathematik, Technische Universität Berlin (jacquier@math.tu-berlin.de), acknowledges financial support from MATHEON.
} 
forward price, to show that it satisfies a small-time large deviation principle. The rate function is computed as FenchelLegendre transform, and they show that this can actually be computed as a standard Legendre transform, which is a simple numerical root-finding exercise. They also establish the corresponding result for implied volatility using well known bounds on the standard Normal distribution function, and they compute the level, the slope and the curvature of the implied volatility in the small-maturity limit around the at-the-money strike, and the answer is consistent with that obtained by formal PDE methods in [24], and probabilistic methods in [8]. Islah[21] derives some closed-form expressions for call options under the SABR model with zero correlation, by integrating over the distribution of the integrated variance, which is known from the work of Matsumoto\&Yor[26] on the Brownian exponential functional, arising by the elegant Bougerol identity.

Gatheral et al.[17] looked at small-time asymptotics for a one-dimensional local volatility model, using Girsanov's theorem and conditioning with a bridge process to derive a small-time expansion for the transition density which holds uniformly in $\mathbb{R}$. They also derive the corresponding expansion for the implied volatility. When the diffusion coefficient is time-dependent, they find that even the leading order term in the expansion requires a small but important modification. For a time-homogenous one-dimension diffusion process $d S_{t}=S_{t} \sigma\left(S_{t}\right) d W_{t}$, they prove the following asymptotic expansion for the implied volatility $\hat{\sigma}(K, t)$ at strike $K$ and time-to-maturity $t$

$$
\hat{\sigma}(K, t)=\hat{\sigma}_{0}(K)+t \frac{\hat{\sigma}_{0}(K)^{3}}{\left(\log \frac{K}{S_{0}}\right)^{2}} \log \frac{\sqrt{\sigma\left(S_{0}\right) \sigma(K)}}{\hat{\sigma}_{0}(K)}+O(t)^{2},
$$

where $\hat{\sigma}_{0}(K)=\left(\frac{1}{\log \frac{K}{S_{0}}} \int_{S_{0}}^{K} \frac{d u}{u \sigma(u)}\right)^{-1}$ is the well known leading order term (see also Busca et al. [1],[2]).

In this article, we add rigour to the arguments of [20],[28],[25] for the following general uncorrelated local-stochastic volatility model for a $\log$ forward price process $X_{t}$

$$
\begin{aligned}
d X_{t} & =-\frac{1}{2} f^{2}\left(Y_{t}\right) d t+f\left(Y_{t}\right) d W_{t}^{1}, \\
d Y_{t} & =\mu\left(Y_{t}\right) d t+\alpha\left(Y_{t}\right) d W_{t}^{2},
\end{aligned}
$$

where $W^{1}$ and $W^{2}$ are independent Brownian motions, subject to certain technical conditions on $f, \mu$ and $\alpha$. We derive a small-time asymptotic for this process (see Theorem 1.1) using the Freidlin-Wentzell theory of large deviations for SDEs, and then converting to the differential geometry problem of computing the shortest distance from a point to a vertical geodesic on $\mathbb{R}^{2}$, furnished with a Riemmanian metric given by the inverse of the diffusion coefficient. This shortest geodesic satisfies a transversality condition, specifically that it comes in perpendicular to the vertical line. We derive the corresponding results for call options and implied volatility in the small-maturity limit in Corollaries 1.2 and 1.4, using Hölder's inequality and a result in [30]. This approach allows us to sidestep the use of viscosity solutions. The cost of doing this is that we have to impose more stringent conditions on $f, \mu$ and $\alpha$ to ensure existence and uniqueness of a classical solution to the variable endpoint calculus of variations problem i.e. for the shortest geodesic. In particular, we only guarantee existence and uniqueness in the region above a certain horizontal line where $f$ has negative second derivative. The manifold has negative sectional curvature in this region. This regime is of practical use when for instance, the maturity of the option is two weeks or less.

In section 3, we show how to extend Theorem 1.1 to a local-stochastic volatility model with zero correlation, using an isometry on the manifold $\mathbb{R}^{2}$ which relates the metric for the local-stochastic volatility model to the metric for the uncorrelated stochastic volatility model. This model can give rise to non-symmetric implied volatility smiles. We also show how we can exogenously choose the local volatility component, so that the model is consistent with the observed limiting behaviour of the implied volatility smile in the short-maturity limit.

In section 4, we compute the level, slope and curvature of the implied volatility in the small-time limit around the at-the-money strike value. In section 5, we introduce the so-called Tanh stochastic volatility model, which satisfies all the conditions of Theorem 1.1.

\subsection{Uncorrelated stochastic volatility}

We work on a model $(\Omega, \mathcal{F}, \mathbb{P})$ with a filtration $\left(\mathcal{F}_{t}\right)_{t \geq 0}$ supporting two Brownian motions which satisfies the usual conditions. 
In this section, we consider a uncorrelated stochastic volatility model for a log forward price process $X_{t}$ defined by the following stochastic differential equations

$$
\begin{aligned}
d X_{t} & =-\frac{1}{2} f^{2}\left(Y_{t}\right) d t+f\left(Y_{t}\right) d W_{t}^{1}, \\
d Y_{t} & =\mu\left(Y_{t}\right) d t+\alpha\left(Y_{t}\right) d W_{t}^{2}
\end{aligned}
$$

with $X_{0}=x_{0} \in \mathbb{R}, Y_{0}=y_{0} \in \mathbb{R}$, where $W^{1}$ and $W^{2}$ are two independent Brownian motions ${ }^{1}$. Assume that $\mu$ is bounded and uniformly Lipschitz continuous, and there exist constants $m_{1}$ and $m_{2}$ such that $0<m_{1} \leq f(y) \wedge \alpha(y) \leq$ $f(y) \vee \alpha(y) \leq m_{2}<\infty, f, \alpha \in C^{\infty},{ }^{2}$. We further assume that $\alpha$ and $f$ are strictly increasing diffeomorphisms from $\mathbb{R}$ to $\left(\alpha_{-\infty}, \alpha_{+\infty}\right)$ and $\left(f_{-\infty}, f_{+\infty}\right)$ respectively, and there exists a $y_{c} \in \mathbb{R}$ such that $f^{\prime \prime}>0, \alpha^{\prime \prime}>0$ for $y<y_{c}$ and $f^{\prime \prime}<0, \alpha^{\prime \prime}<0$ for $y>y_{c}$.

The conditions above imply that $f(y) \rightarrow f_{ \pm \infty}, \alpha(y) \rightarrow \alpha_{ \pm \infty}$ as $y \rightarrow \pm \infty$ (which ensures that $\pm \infty$ are natural boundaries for the $Y$ process) and that $f^{\prime}>0, \alpha^{\prime}>0$, and $f^{\prime} \vee \alpha^{\prime}<C<\infty$ for some constant $C$, so $f$ and $\alpha$ are also uniformly Lipschitz continuous.

We also impose the linear growth condition that the function

$$
u \mapsto \frac{\alpha\left(f^{-1}(u)\right)}{u}
$$

is non-increasing ${ }^{3}$.

In section 2 , we derive the following result which describes the asymptotic behaviour of $X_{t}-x_{0}$ as $t \rightarrow 0$.

Theorem 1.1 We have the following small-time behaviour for $X_{t}$

$$
-\lim _{t \rightarrow 0} t \log \mathbb{P}\left(X_{t}-x_{0}>x_{1}\right)=\frac{1}{2} d\left(x_{1}\right)^{2}
$$

for $x_{1}>0$, where $d\left(x_{1}\right)$ represents the distance from $\left(0, y_{0}\right)$ to the line $\left\{x=x_{1}\right\}$ on the Riemannian manifold $M=\mathbb{R}^{2}$, with metric $\boldsymbol{g}$ whose coefficients are given by $g_{11}=\frac{1}{f(y)^{2}}, g_{12}=0, g_{22}=\frac{1}{\alpha(y)^{2}}$. If $y_{0}>y_{c}$, then there is a unique distance-minimizing geodesic $\gamma^{*}$ from $\left(0, y_{0}\right)$ to the line $\left\{x=x_{1}\right\}$, and $\gamma^{*}$ is perpendicular to the $y$-axis at the point $\left(x_{1}, y_{1}^{*}\left(x_{1}\right)\right)$ where it intersects with the line $\left\{x=x_{1}\right\}$. We can calculate $d\left(x_{1}\right)$ explicitly using the following two equations

$$
\begin{aligned}
d\left(x_{1}\right) & =f\left(y_{1}^{*}\left(x_{1}\right)\right) \int_{y_{0}}^{y_{1}^{*}\left(x_{1}\right)} \frac{d y}{\alpha(y) \sqrt{f\left(y_{1}^{*}\left(x_{1}\right)\right)^{2}-f(y)^{2}}}, \\
x_{1} & =\int_{y_{0}}^{y_{1}^{*}\left(x_{1}\right)} \frac{f(y)^{2} d y}{\alpha(y) \sqrt{f\left(y_{1}^{*}\left(x_{1}\right)\right)^{2}-f(y)^{2}}} .
\end{aligned}
$$

We first solve for the unique $y_{1}\left(x_{1}^{*}\right)$ associated with $x_{1}$ in (6), and then substitute this $y_{1}^{*}$ value into (5) to compute the distance $d\left(x_{1}\right)$.

Remark 1.1 Similar equations to (5) and (6) are derived on page 161 in Henry-Labordère[20], but without proving that $y_{1}\left(x_{1}^{*}\right)$ is unique and without the geometrical interpretation. Explicit calculations for the SABR model appear in Eq 6.47 in [20]. At leading order, the Freidlin-Wentzell large deviations approach obviates the need for small-time estimates for the heat kernel and the effective local volatility as in [20], which was not rigorous.

Remark 1.2 As is typical in the Freidlin-Wentzell theory of large deviations for SDEs in the small-time limit, the drift term $\mu$ does not affect the rate function. Note that the value of $\alpha(y)$ and $f(y)$ for $y<y_{0}$ has no effect on the small-time asymptotics.

Remark 1.3 Note that the Black-Scholes model where $f$ is constant is a degenerate special case of the model in (2), which is not covered by Theorem 3.1. See [14] for small-time asymptotics for Black-Scholes.

\footnotetext{
${ }^{1}$ This model is similar to the type of model used in [15] where the volatility process is bounded away from zero.

2 The existence and uniqueness of a strong solution for the $Y$ process is standard, and the $X$ process can be expressed as a stochastic integral of the $Y$ process, so $X$ is also well defined. $f$ is bounded, so the Novikov condition is satisfied and $e^{X_{t}}$ is a martingale.

${ }^{3}$ Note that this condition is trivially satisfied if $\alpha(y)=\xi f(y)$ for some $\xi>0$, or if $\alpha$ is just a positive constant.
} 
A useful corollary of this result is the following rare event estimate for pricing out-of-the-money put/call options of small maturity, derived in Section 2.2.

Corollary 1.2 For the stochastic volatility model in (2), we have the following small-time behaviour for out-of-themoney put/call options on $S_{t}=e^{X_{t}}$

$$
\begin{aligned}
& -\lim _{t \rightarrow 0} t \log \mathbb{E}\left(S_{t}-K\right)^{+}=\frac{1}{2} d(x)^{2} \quad(x \geq 0), \\
& -\lim _{t \rightarrow 0} t \log \mathbb{E}\left(K-S_{t}\right)^{+}=\frac{1}{2} d(x)^{2} \quad(x \leq 0),
\end{aligned}
$$

where $x=\log \frac{K}{S_{0}}$ is the log-moneyness.

Remark $1.4 d(0)=0$, so these estimates are very crude for at-the-money options.

Using the put-call parity, we can re-write Corollary 1.2 as follows.

Corollary 1.3 For all $x \in \mathbb{R}$

$$
\lim _{t \rightarrow 0} t \log \left(\mathbb{E}\left(S_{t}-K\right)^{+}-\left(S_{0}-K\right)^{+}\right)=\lim _{t \rightarrow 0} t \log \left(\mathbb{E}\left(K-S_{t}\right)^{+}-\left(K-S_{0}\right)^{+}\right)=\frac{1}{2} d(x)^{2},
$$

where $x=\log \frac{K}{S_{0}}$.

Moreover, the asymptotic implied volatility can be computed as follows:

Corollary 1.4 We have the following asymptotic behaviour for the implied volatility $\sigma_{t}(x)$ of an out-of-the-money European call option on $S_{t}=e^{X_{t}}$, with strike $K=S_{0} e^{x}(x>0)$, as $t \rightarrow 0$

$$
I(x)=\lim _{t \rightarrow 0} \sigma_{t}(x)=x / d(x) .
$$

Proof. Follows from Corollary 1.2 and Theorem 5.1 in [30].

\section{Proof of main results}

\subsection{Small-time behaviour of probabilities}

In this subsection, we prove Theorem 3.1 in four steps: we start by proving a small-time large deviation principle for the uncorrelated stochastic volatility model in (2) using Freidlin-Wentzell theory. We then show that computing the rate function is tantamount to finding the shortest distance to a vertical line under the Riemmanian metric induced by the diffusion coefficient of the SDE. The third part, more involved, integrates the geodesic equations and establishes uniqueness for the shortest geodesic.

\section{Proof.}

\section{Part 1: Applying Freidlin-Wentzell theory to the uncorrelated stochastic volatility model.}

We can set $X_{0}=0$ without loss of generality as $X_{t}-x_{0}$ is independent of $x_{0}$, because the SDEs have no $x$ dependence. Let $X_{t}^{\epsilon}$ and $Y_{t}^{\epsilon}$ denote the unique strong solution of the stochastic differential equation

$$
\begin{aligned}
& d X_{t}^{\epsilon}=-\frac{1}{2} \epsilon f\left(Y_{t}^{\epsilon}\right)^{2} d t+\sqrt{\epsilon} f\left(Y_{t}^{\epsilon}\right) d W_{t}^{1}, \\
& d Y_{t}^{\epsilon}=\epsilon \mu\left(Y_{t}^{\epsilon}\right) d t+\sqrt{\epsilon} \alpha\left(Y_{t}^{\epsilon}\right) d W_{t}^{2} .
\end{aligned}
$$

$X_{0}^{\epsilon}=0, Y_{0}=y_{0} \in \mathbb{R}$. Then $X_{t}^{\epsilon}$ and $Y_{t}^{\epsilon}$ have the same drift and diffusion coefficient terms as $X_{\epsilon t}$ and $Y_{\epsilon t}$ respectively, so they have the same law. It is well known (see e.g. Theorem 6.3 in Varadhan[33]) that $\left\{\left(X^{\epsilon}(),. Y^{\epsilon}().\right)\right\}$ satisfies a large deviation principle (LDP) in $C\left([0,1], \mathbb{R}^{2}\right)$ as $\epsilon \rightarrow 0$, with lower semicontinuous rate function

$$
I(x, y)=\frac{1}{2} \int_{0}^{1}\left(\frac{1}{f(y(t))^{2}} \dot{x}^{2}+\frac{1}{\alpha(y(t))^{2}} \dot{y}^{2}\right) d t .
$$


By the contraction principle (Theorem 4.2.1, page 110 in [6]), $X_{1}^{\epsilon}$ (and hence $X_{\epsilon}$ ) satisfy a small-time LDP with the lower semi-continuous rate function $\iota: \mathbb{R} \rightarrow[0, \infty]$ defined by

$$
\iota\left(x_{1}\right)=\frac{1}{2} \inf _{(x, y) \in C_{\left(0, y_{0}\right)}\left([0,1], \mathbb{R}^{2}\right): x(1)=x_{1}} \int_{0}^{1}\left(\frac{1}{f(y(t))^{2}} \dot{x}^{2}+\frac{1}{\alpha(y(t))^{2}} \dot{y}^{2}\right) d t .
$$

Thus, by the definition of the large deviation principle, it follows that

$$
-\inf _{x: x>x_{1}} \iota\left(x_{1}\right) \leq \liminf _{\epsilon \rightarrow 0} \epsilon \log \mathbb{P}\left(X_{\epsilon}-x_{0}>x_{1}\right) \leq \limsup _{\epsilon \rightarrow 0} \epsilon \log \mathbb{P}\left(X_{\epsilon}-x_{0} \geq x_{1}\right) \leq-\inf _{x: x \geq x_{1}} \iota\left(x_{1}\right) .
$$

We will show that $\iota\left(x_{1}\right)$ is continuous as a function of $x_{1}$, so the liminf and lim sup here can actually be replaced by a true limit.

Part 2: Converting from a large deviations problem to a differential geometry problem: the distance from a point to a geodesic.

$\iota\left(x_{1}\right)$ is obtained as the solution to a variable endpoint calculus of variations problem, which is equivalent to the differential geometry problem of finding the infimum of the Energy functional $E(x(),. y())=.I(x(),. y()$.$) over$ all piecewise differentiable curves joining $\left(0, y_{0}\right)$ to the line $\left\{x=x_{1}\right\}$ on the manifold $M=\mathbb{R}^{2}$, furnished with the Riemmanian metric $\mathbf{g}$ whose coefficients are given by

$$
g_{11}=\frac{1}{f(y)^{2}}, g_{12}=0, g_{22}=\frac{1}{\alpha(y)^{2}} .
$$

Without loss of generality, we assume that $x_{1}>0$, because the metric is independent of $x$, so the shortest geodesic to $\left\{x=-x_{1}\right\}$ is just the reflection of the shortest geodesic to $\left\{x=x_{1}\right\}$ about the $y$-axis.

By the Hopf-Rinow theorem (Theorem 2.8, page 147 in [4]), a Riemmanian manifold is complete as a metric space if and only if it is geodesically complete, which is true if and only if for any two points $p$ and $q$ in $M$, there exists a distance-minimizing geodesic $\gamma$ joining $p$ and $q$ i.e. the length $l(\gamma)$ of $\gamma$ under the metric $\mathbf{g}$ satisfies $l(\gamma)=d(p, q)$, where $d($.$) is the distance function for M$ (see Definition 2.4, page 146 in [4]). $\mathbb{R}^{2}$ is complete ${ }^{4}$, so using the bounds on $f$, we can easily compare Cauchy sequences on $M$ to $\mathbb{R}^{2}$, and verify that $M$ is complete as a metric space. Thus, by Lemma 2.3, page 194 in [4]), we have that

$$
I(\gamma)=\frac{1}{2} l(\gamma)^{2}
$$

and $\gamma$ not only minimizes the distance but also minimizes the Energy functional for continuous curves joining $p$ to $q$.

\section{Part 3: Integrating the geodesic equations.}

The non-zero Christoffel symbols for the metric $\mathbf{g}$ are given by

$$
\Gamma_{11}^{2}=f(y)^{-3} \alpha(y)^{2} f^{\prime}(y), \quad \Gamma_{21}^{1}=\Gamma_{12}^{1}=-f(y)^{-1} f^{\prime}(y), \quad \Gamma_{22}^{2}=-\alpha(y)^{-1} \alpha^{\prime}(y)
$$

(see page 161 in [4]). Thus the geodesic equations $\ddot{x}^{\lambda}+\Gamma_{\mu \nu}^{\lambda} \dot{x}^{\mu} \dot{x}^{\nu}=0$ take the form

$$
\begin{aligned}
\ddot{x}-2 f(y)^{-1} f^{\prime}(y) \dot{x} \dot{y} & =0, \\
\ddot{y}+f(y)^{-3} \alpha(y)^{2} f^{\prime}(y) \dot{x}^{2}-\alpha(y)^{-1} \alpha^{\prime}(y) \dot{y}^{2} & =0 .
\end{aligned}
$$

If we substitute $y(t)=$ constant into the geodesic equations, we find that $\dot{x}=0$ i.e. $x$ is also constant. This means that we cannot have horizontal geodesics. We can divide (14) by $f(y)^{2}$, and integrate to obtain

$$
\dot{x}=K f(y)^{2},
$$

for some constant $K$. In this article, we will only need to consider geodesics for which $\dot{x}>0$, so we can assume $K>0$. Note that $0<\dot{x}<\infty$, so that $\dot{y}=0$ if and only if $d y / d x=0$. If $\dot{y} \neq 0$, we can multiply (15) by $2 \dot{y} / \alpha(y)^{2}$ to obtain

$$
\begin{aligned}
2\left[\frac{1}{\alpha(y)^{2}} \ddot{y} \dot{y}+f(y)^{-3} \dot{x}^{2} f^{\prime}(y) \dot{y}-\alpha(y)^{-3} \alpha^{\prime}(y) \dot{y}^{2} \dot{y}\right] & =2\left[\frac{1}{\alpha(y)^{2}} \ddot{y} \dot{y}+K^{2} f^{\prime}(y) f(y) \dot{y}-\alpha(y)^{-3} \alpha^{\prime}(y) \dot{y}^{2} \dot{y}\right] \\
& =\frac{d}{d t}\left(\frac{1}{\alpha(y)^{2}} \dot{y}^{2}+K^{2} f(y)^{2}\right)=0 .
\end{aligned}
$$

\footnotetext{
${ }^{4}$ Note that the metric associated with the well known Heston model for unit volatility-of-variance $d s^{2}=y^{-1}\left(d x^{2}+d y^{2}\right)$ is not complete, because geodesics can escape to the $x$-axis in finite time. Interestingly, [19] and[23] point out that this space has infinite curvature at $y=0$, similar to a black hole in the Schwarzschild metric in general relativity.
} 
Thus

$$
K^{2} f(y)^{2}+\frac{1}{\alpha(y)^{2}} \dot{y}^{2}=E,
$$

for some constant $E$, or (taking the positive square root) and setting $c:=E / K^{2}$, we have

$$
\frac{d t}{d y}=\alpha(y)^{-1}\left(E-K^{2} f(y)^{2}\right)^{-1 / 2}=K^{-1} \alpha(y)^{-1}\left(c-f(y)^{2}\right)^{-1 / 2}
$$

for $f(y)^{2}<c$. We can integrate this autonomous ODE directly to obtain

$$
t(y)=\frac{1}{K} \int_{y_{0}}^{y} \frac{d u}{\alpha(y) \sqrt{c-f(u)^{2}}} .
$$

Now assume that $c$ is chosen such that $f\left(y_{0}\right)^{2}<c<f_{+\infty}^{2}$, so that $\dot{y} \in(0, \infty)$ at $t=0 .{ }^{5}$ Then $\dot{y}=0$ at

$$
y^{* *}=f^{-1}(\sqrt{c})>y_{0}
$$

and $\dot{y}>0$ for $y<y^{* *}$. Thus $t(y)$ is invertible for $y<y^{* *}$. The length of this geodesic is given by

$$
d=\int_{0}^{t(y)}|\dot{\gamma}| d t=\frac{\sqrt{E}}{K} \int_{y_{0}}^{y} \frac{d u}{\alpha(u) \sqrt{c-f(u)^{2}}}=\sqrt{c} \int_{y_{0}}^{y} \frac{d u}{\alpha(u) \sqrt{c-f(u)^{2}}} .
$$

For $t>t^{* *}=t\left(y^{* *}\right)$, we have to consider the negative square root i.e. the solution to the ODE $d t / d y=-\left(\alpha(y) \sqrt{E-K^{2} f(y)^{2}}\right)^{-1}$, and the solution is given by

$$
t(y)=\frac{1}{K} \int_{y_{0}}^{f^{-1}(\sqrt{c})} \frac{d y}{\alpha(y) \sqrt{c-f(y)^{2}}}+\frac{1}{K} \int_{y}^{f^{-1}(\sqrt{c})} \frac{d y}{\alpha(y) \sqrt{c-f(y)^{2}}},
$$

for $t>t^{* *}$, and we see that the geodesic bends back on itself. The spurious solution given by the horizontal line $x(t)=K^{2} f^{2}\left(y^{* *}\right) t$ and $y(t)=y^{* *}$ also satisfies (16) and (18), but it fails to satisfy the original geodesic equations (14) and (15), and thus is not a true geodesic. This is an artefact of multiplying by $\dot{y}$ when we integrated the geodesic equations. Thus we see that for a given $K$ and $E$, there is only solution to (18) which is a valid geodesic. By Corollary 3.9 on page 73 in [4], we know that if any piecewise differentiable curve is minimizing, then it has to be a geodesic. Since we have established existence and uniqueness for the geodesics, we see that the inverse of the solution $t(y)$ in (19) is the $y$-component of a distance-minimizing geodesic. Using (18), we can compute the $x$-component $x(t)$ by integrating (16) explicitly to obtain

$$
x(t)=K \int_{0}^{t} f(y(s))^{2} d s=\int_{y_{0}}^{y(t)} \frac{f^{2}(y) d y}{\alpha(y) \sqrt{c-f(y)^{2}}},
$$

for $t \leq t^{* *}$. From (20), we see that if the following condition is satisfied

$$
0<x_{1}<\int_{y_{0}}^{y_{1}} \frac{f^{2}(y) d y}{\alpha(y) \sqrt{f\left(y_{1}\right)^{2}-f(y)^{2}}},
$$

for a given $x_{1}>0$ and $y_{1}>y_{0}$, then there is a unique $c \in\left(f\left(y_{1}\right)^{2}, \infty\right)$ which satisfies

$$
x_{1}=\int_{y_{0}}^{y_{1}} \frac{f^{2}(y) d y}{\alpha(y) \sqrt{c-f(y)^{2}}}
$$

because $x_{1}$ is a continuous, strictly monotonically decreasing function of $c$. This means that we can find a unique geodesic joining $\left(0, y_{0}\right)$ to $\left(x_{1}, y_{1}\right)$ which does not bend back on itself.

We now return to the variable endpoint problem of finding a minimizing geodesic from the point $p=\left(0, y_{0}\right)$ to the vertical line $\left\{x=x_{1}\right\}, x_{1}>0$. From the bounds on $f$ and $\alpha$, we see that the distance from $\left(0, y_{0}\right)$ to $\left(x_{1}, y_{1}\right)$ tends to infinity as $y_{1} \rightarrow \pm \infty$, so any minimising geodesic $\gamma^{*}$ for the variable endpoint problem must be finite at $x=x_{1}$. Now consider a variation ${ }^{6} f(s, t), f:(-\epsilon, \epsilon) \times[0, a] \rightarrow M$ of any geodesic $\gamma$ which starts at $\left(0, y_{0}\right)$ and also passes through

\footnotetext{
${ }^{5}$ For the variable endpoint problem that we need to solve, we can restrict attention to geodesics with $\dot{y}(0)>0$, because any geodesic starting from $\left(0, y_{0}\right)$ with $\dot{y}(0)<0$ which also passes through the line $\left\{x=x_{1}\right\}, x_{1}>0$, will be longer than the segment of the horizontal line $\left\{y=y_{0}\right\}$ between $x=0$ and $x=x_{1}$, because the two functions $g_{11}=\frac{1}{f(y)^{2}}$, and $g_{22}=\frac{1}{\alpha(y)^{2}}$ are both strictly decreasing in $y$.

${ }^{6}$ see [4] for a definition of a variation.
} 
the line $\left\{x=x_{1}\right\}$, which satisfies $f(s, 0)=\left(0, y_{0}\right)$ and the $x$-component of $f(s, a)=x_{1}$ for all $\left.s \in(-\epsilon, \epsilon)\right)$. Then, from Proposition 2.4, page 195 in [4], we have the following expression for the first variation of the Energy functional as a function of $s$

$$
E^{\prime}(0)=\mathbf{g}\left(\left.\frac{\partial f}{\partial s}\right|_{s=0, t=a}, \frac{d \gamma}{d t}(a)\right)
$$

${ }^{7}$ Thus, we see that for any geodesic which is not perpendicular to $\left\{x=x_{1}\right\}$ when it passes through this line, we can make a small perturbation to the geodesic, and obtain a shorter curve. In other words, we see that a necessary condition for an extremal curve $\gamma^{*}(t)=\left(x^{*}(t), y^{*}(t)\right)$ for the variable endpoint problem is given by the transversality condition

$$
\mathbf{g}\left((0,1), \frac{d \gamma^{*}}{d t}\right)=\left.\frac{1}{\alpha^{2}\left(y_{1}^{*}\right)} \frac{d y^{*}}{d t}\right|_{\left(x_{1}, y_{1}^{*}\right)}=\left.0 \Longrightarrow \frac{d y^{*}}{d t}\right|_{\left(x_{1}, y_{1}^{*}\right)}=0
$$

where $y_{1}^{*}$ is the $y$-value where $\gamma^{*}$ passes through the line $\left\{x=x_{1}\right\}$. From (18), we see that if we set $c=c^{*}$, where

$$
c^{*}=f\left(y_{1}\right)^{2},
$$

then $\dot{y}=0$ at $y=y_{1}$. From (20), we see that this turning point occurs at the $x$-value

$$
x^{* *}=\int_{y_{0}}^{f^{-1}\left(\sqrt{c^{*}}\right)} \frac{f(y)^{2} d y}{\alpha(y) \sqrt{c^{*}-f(y)^{2}}} .
$$

Now let $z=c^{*}-f(y)^{2}$. Then

$$
\frac{d z}{d y}=-2 f(y) f^{\prime}(y)=-2 \sqrt{c^{*}-z} f^{\prime}\left(f^{-1}\left(\sqrt{c^{*}-z}\right)\right),
$$

and we can re-write (22) as

$$
\begin{aligned}
x^{* *}=x^{* *}\left(c^{*}\right) & =\int_{0}^{c^{*}-f\left(y_{0}\right)^{2}} \frac{c^{*}-z}{\alpha\left(f^{-1}\left(\sqrt{c^{*}-z}\right)\right) \sqrt{z}} \cdot \frac{d z}{2 \sqrt{c^{*}-z} f^{\prime}\left(f^{-1}\left(\sqrt{c^{*}-z}\right)\right)} \\
& =\int_{0}^{c^{*}-f\left(y_{0}\right)^{2}} \frac{\sqrt{c^{*}-z}}{\alpha\left(f^{-1}\left(\sqrt{c^{*}-z}\right)\right) \sqrt{z}} \cdot \frac{d z}{2 f^{\prime}\left(f^{-1}\left(\sqrt{c^{*}-z}\right)\right)}
\end{aligned}
$$

By the condition in (3), and the fact that $f^{\prime}$ is strictly monotonically decreasing for $y>y_{c}$, we see that the integrand is non-decreasing in $c^{*}$, and

$$
\frac{d x^{* *}}{d c^{*}}>0 .
$$

Moreover, from (22) we see that $x^{* *}($.$) maps \left(f\left(y_{0}\right)^{2}, f_{\infty}^{2}\right)$ into $(0, \infty)$. Thus $x^{* *}\left(c^{*}\right)$ is a differentiable, strictly increasing function of $c^{*}$, with $x^{* *}\left(f\left(y_{0}\right)^{2}\right)=0$ and $x^{* *}\left(c^{*}\right) \rightarrow \infty$ as $c^{*} \rightarrow f_{\infty}^{2}$, so we cannot have two distinct geodesics (with two different $c^{*}$ values) which both start at $\left(0, y_{0}\right)$ and are both perpendicular to the $y$-axis at $\left\{x=x_{1}\right\}$. From (21) and (24), we also have that

$$
\frac{d y_{1}^{*}\left(x_{1}\right)}{d x_{1}}=\left(\frac{d x^{* *}}{d c^{*}} \cdot \frac{d c^{*}}{d y}\right)^{-1}=\left(2 \frac{d x^{* *}}{d c^{*}} f\left(y_{1}^{*}\right) f^{\prime}\left(y_{1}^{*}\right)\right)^{-1}>0 .
$$

Thus for any $x_{1}>0$, we conclude that there is a unique geodesic from $\left(0, y_{0}\right)$ to the line $\left\{x=x_{1}\right\}$ which is perpendicular to the $y$-axis at $\left\{x=x_{1}\right\}$, and this is the shortest geodesic to this vertical line. Let $y_{1}^{*}\left(x_{1}\right)$ denote the $y$-value where this geodesic intercepts $\left\{x=x_{1}\right\}$. To calculate the length of the shortest geodesic, we need the following two equations

$$
\begin{aligned}
d\left(x_{1}\right) & =f\left(y_{1}^{*}\left(x_{1}\right)\right) \int_{y_{0}}^{y_{1}^{*}\left(x_{1}\right)} \frac{d y}{\alpha(y) \sqrt{f\left(y_{1}^{*}\left(x_{1}\right)\right)^{2}-f(y)^{2}}}, \\
x_{1} & =\int_{y_{0}}^{y_{1}^{*}\left(x_{1}\right)} \frac{f^{2}(y) d y}{\alpha(y) \sqrt{f\left(y_{1}^{*}\left(x_{1}\right)\right)^{2}-f(y)^{2}}}
\end{aligned}
$$

(Recall that $\left.c^{*}=f\left(y_{1}^{*}\left(x_{1}\right)\right)^{2}\right)$. We first solve for the unique $y_{1}\left(x_{1}^{*}\right)$ associated with $x_{1}$ in (27), and then substitute this into (26) to compute the distance. Clearly $d\left(x_{1}\right)$ is increasing in $x_{1}$. Moreover, the distance function $d(p, q)$ for $M$ is continuous (see Corollary 2.7 in chapter 7 of [4]). Thus, for any $\epsilon>0$, if we choose $\delta=f_{-\infty} \epsilon$, then if $\left|x^{\prime}-x\right|<\delta$, we have

$$
\left|d\left(x^{\prime}\right)-d(x)\right| \leq \delta / f_{-\infty}=\epsilon,
$$

so we see that $d\left(x_{1}\right)$ is increasing and continuous, and the result follows from the definition of the large deviation principle with the rate function $\iota\left(x_{1}\right)=\frac{1}{2} d\left(x_{1}\right)^{2}$.

\footnotetext{
${ }^{7}$ Note that the Energy functional $E$ as we define it here is one-half of the $E$ that is used in [4].
} 
Remark 2.1 For a general stochastic volatility model with non-zero correlation, i.e. $d\left\langle W_{t}^{1}, W_{t}^{2}\right\rangle=\rho d t$, the shortest geodesic $\gamma^{*}$ also comes in perpendicular to the line $\left\{x=x_{1}\right\}$ under the metric $\mathbf{g}$ associated with the correlated model (see also Exercise 6 on page 208 in [4]). In this case the coefficients of the metric are $g_{11}=\frac{1}{f(y)^{2} \bar{\rho}^{2}}, g_{12}=g_{21}=$ $-\frac{\rho}{f(y) \alpha(y) \rho^{2}}, g_{22}=\frac{1}{\alpha(y)^{2} \bar{\rho}^{2}}$ where $\bar{\rho}=\sqrt{1-\rho^{2}}$, and the transversality condition on the vertical line $\left\{x=x_{1}\right\}$ is

$$
\left.\mathbf{g}\left((0,1),\left(\frac{d x}{d t}, \frac{d y}{d t}\right)\right)\right|_{\left(x_{1}, y_{1}^{*}\right)}=\left.\left(g_{21} \frac{d x}{d t}+g_{22} \frac{d y}{d t}\right)\right|_{\left(x_{1}, y_{1}^{*}\right)}=0,
$$

so $\left.\frac{d y}{d x}\right|_{\left(x_{1}, y_{1}^{*}\right)}=-g_{21} / g_{22}=\rho \alpha\left(y_{1}^{*}\right) / f\left(y_{1}^{*}\right)$, which is negative if $\rho<0$, which means that the minimizing geodesic must bend back if $\left.\frac{d y}{d x}\right|_{\left(0, y_{0}\right)}>0$, which will be the case for $x_{1}$ sufficiently large.

\subsection{Small-time behaviour of European options}

Here we prove Corollary 1.2:

Proof. (i) We first deal with the lower bound. For any $\delta>0$, we have

$$
\mathbb{E}\left(S_{t}-K\right)^{+} \geq \delta \mathbb{P}\left(S_{t}>K+\delta\right) .
$$

Then by Theorem 1.1 we have that

$$
\liminf _{t \rightarrow 0} t \log \mathbb{E}\left(S_{t}-K\right)^{+} \geq \liminf _{t \rightarrow 0}\left[t \log \delta+t \log \mathbb{P}\left(S_{t}>K+\delta\right)\right] \geq-\frac{1}{2} d\left(\log \frac{K+\delta}{S_{0}}\right)^{2},
$$

Take $\delta \rightarrow 0+$. By continuity of $d(x)$, we have the desired lower bound.

(ii) To show the desired upper bound, we note that for any $p, q>1$ with $p^{-1}+q^{-1}=1$, we have

$$
\mathbb{E}\left(S_{t}-K\right)^{+}=\mathbb{E}\left(\left(S_{t}-K\right)^{+} 1_{S_{t} \geq K}\right) \leq\left[\mathbb{E}\left(\left(S_{t}-K\right)^{+}\right)^{p}\right]^{1 / p} \mathbb{P}\left(S_{t} \geq K\right)^{1 / q} .
$$

Thus

$$
t \log \mathbb{E}\left(S_{t}-K\right)^{+} \leq \frac{t}{p} \log \mathbb{E}\left(\left(S_{t}-K\right)^{+}\right)^{p}+t\left(1-\frac{1}{p}\right) \log \mathbb{P}\left(S_{t} \geq K\right) \leq \frac{t}{p} \log \mathbb{E}\left(S_{t}^{p}\right)+t\left(1-\frac{1}{p}\right) \log \mathbb{P}\left(S_{t} \geq K\right) .
$$

By conditioning on $T(t)=\int_{0}^{t} f\left(Y_{s}\right)^{2} d s$, we can write $X_{t}$ as an arithmetic Brownian motion evaluated at a bounded independent stochastic time change

$$
X_{t}-x_{0}=-\frac{1}{2} T(t)+W_{T(t)}^{1} .
$$

Thus

$$
e^{\frac{1}{2}\left(p^{2}-p\right) m_{1}^{2} t} \leq \mathbb{E}\left(\left(\frac{S_{t}}{S_{0}}\right)^{p}\right)=\mathbb{E}\left(e^{\frac{1}{2}\left(p^{2}-p\right) \int_{0}^{t} f^{2}\left(Y_{s}\right) d s}\right) \leq e^{\frac{1}{2}\left(p^{2}-p\right) m_{2}^{2} t} .
$$

and $\mathbb{E}\left(S_{t}^{p}\right) \rightarrow S_{0}^{p}$ as $t \rightarrow 0$. If we then take $\lim _{p \rightarrow \infty} \lim _{\sup _{t \rightarrow 0}}$ on both sides of (31), we have (by Theorem 1.1) the upper bound

$$
\limsup _{t \rightarrow 0} t \log \mathbb{E}\left(S_{t}-K\right)^{+} \leq-\frac{1}{2} d\left(\log \frac{K}{S_{0}}\right)^{2} .
$$

\section{Local-stochastic volatility}

In this section we extend the results of the previous section to incorporate a local volatility component, so the model can now give rise to non-symmetric implied volatility smiles.

Theorem 3.1 Consider the following local-stochastic volatility model for a log forward price process $X_{t}$

$$
\begin{aligned}
d X_{t} & =-\frac{1}{2} \sigma\left(X_{t}\right)^{2} f\left(Y_{t}\right)^{2} d t+\sigma\left(X_{t}\right) f\left(Y_{t}\right) d W_{t}^{1}, \\
d Y_{t} & =\mu\left(Y_{t}\right) d t+\alpha\left(Y_{t}\right) d W_{t}^{2},
\end{aligned}
$$


with $X_{0}=x_{0}, Y_{0}=y_{0}>y_{c}>0, f, \mu, \alpha$ as in Theorem 1.1, $\sigma$ uniformly Lipschitz continuous, $0<\sigma_{\min }<\sigma(x)<$ $\sigma_{\max }<\infty$ and $W^{1}$ and $W^{2}$ independent Brownian motions. ${ }^{8}$ Then we have the following small-time behaviour for the implied volatility

$$
I\left(x_{1}\right)=\lim _{t \rightarrow 0} \sigma_{t}\left(x_{1}\right)=\frac{x_{1}-x_{0}}{d^{\operatorname{loc}}\left(x_{0}, x_{1}\right)},
$$

where $x_{1}=\log K$ is the log-strike,

$$
d^{\mathrm{loc}}\left(x_{0}, x_{1}\right)=d\left(\int_{x_{0}}^{x_{1}} \frac{d u}{\sigma(u)}\right)
$$

and $d($.$) is given in (5).$

Proof. The Riemmanian metric induced by the diffusion coefficient for this model is given by $\mathbf{g}^{\text {loc }}(.,$.$) , with$ coefficients

$$
g_{11}^{\text {loc }}=\sigma(x)^{-2} \frac{1}{f(y)^{2}}, \quad g_{12}^{\text {loc }}=0, \quad \text { and } \quad g_{22}^{\text {loc }}=\frac{1}{\alpha(y)^{2}} .
$$

Let $d^{\text {loc }}\left(x_{0}, x_{1}\right)$ denote the distance from $\left(x_{0}, y_{0}\right)$ to the line $\left\{x=x_{1}\right\}$ under the metric $\mathbf{g}^{\text {loc }}(.,$.$) . Now consider$ the diffeomorphism $\Psi: \mathbb{R}^{2} \rightarrow \mathbb{R}^{2}, \Psi(x, y)=\left(q^{-1}(x), y\right)$, where $q(x)=\int_{x_{0}}^{x} \sigma(u)^{-1} d u$. Then $\Psi$ induces a Riemmanian structure $\mathbf{g}$ on $\mathbb{R}^{2}$, with

$$
\mathbf{g}(u, v)_{p}=\mathbf{g}^{\mathrm{loc}}(d \Psi(u), d \Psi(v))_{\Psi(p)}=\frac{1}{f(y)^{2}} u_{1} v_{1}+\frac{1}{\alpha(y)^{2}} u_{2} v_{2},
$$

where $u:=\left(u_{1}, u_{2}\right)$ and $v:=\left(v_{1}, v_{2}\right)$, at a point $p \in \mathbb{R}^{2}$, so we see that $\mathbf{g}$ is the same metric that appears in (12), and the mapping $\Psi$ is an isometry (see example 2.5, page 39 in [4]). Thus, there is a unique distance minimizing geodesic joining $\left(x_{0}, y_{0}\right)$ to the line $\left\{x=x_{1}\right\}$ under the metric $\mathbf{g}^{\text {loc }}$, whose length $d^{\text {loc }}\left(x_{0}, x_{1}\right)=d\left(q\left(x_{1}\right)\right)$, where $d($. is the same distance function that appears in (4), and $d^{\mathrm{loc}}\left(x_{0}, x_{1}\right)$ is continuous as a function of $x_{1}$. Moreover, by the Freidlin-Wentzell theorem and the contraction principle, we have that

$$
-\lim _{t \rightarrow 0} t \log \mathbb{P}\left(X_{t}>x_{1}\right)=\frac{1}{2} d^{\mathrm{loc}}\left(x_{0}, x_{1}\right)^{2}=\frac{1}{2} d\left(q\left(x_{1}\right)\right)^{2},
$$

and the result follows using a similar argument to Corollary 1.4.

\subsection{Calibrating $\sigma(x)$ to the observed small-maturity smile}

Note that we can use (36) and (37) as follows

$$
q\left(x_{1}\right)=d^{-1}\left(\frac{x_{1}-x_{0}}{I\left(x_{1}\right)}\right), \quad \sigma(x)=\frac{1}{q^{\prime}(x)}
$$

to back out a $\sigma(x)$ function so that the model is consistent with the observed behaviour of the implied volatility smile in the small-maturity limit i.e. the observed $I\left(x_{1}\right)=\lim _{t \rightarrow 0} \sigma_{t}\left(x_{1}\right)$, if this limit exists for all $x_{1}$, as long as the $\sigma$ that we extract is uniformly Lipschitz continuous and bounded from above and below by positive constants.

\section{The level, slope and convexity of the small-time implied volatility at- the-money}

Theorem 4.1 For the general local-stochastic volatility model in Theorem 3.1, the asymptotic implied volatility I $\left(x_{1}\right)$ has the following expansion around the at-the-money log strike $x_{1}=x_{0}$

$$
\begin{aligned}
I\left(x_{1}\right) & =\sigma\left(x_{0}\right) f\left(y_{0}\right)+\frac{1}{2} \sigma^{\prime}\left(x_{0}\right) f\left(y_{0}\right)\left(x_{1}-x_{0}\right) \\
& +\left(-\frac{1}{3} \frac{\sigma^{\prime}\left(x_{0}\right)^{2}}{\sigma\left(x_{0}\right)}+\frac{1}{6} \sigma^{\prime \prime}\left(x_{0}\right)+\frac{1}{6} \frac{f^{\prime}\left(y_{0}\right)^{2} \alpha\left(y_{0}\right)^{2}}{f\left(y_{0}\right)^{4} \sigma\left(x_{0}\right)}+\frac{1}{4} \frac{\sigma^{\prime}\left(x_{0}\right)^{2}}{\sigma\left(x_{0}\right)}\right) f\left(y_{0}\right)\left(x_{1}-x_{0}\right)^{2}+O\left(x_{1}-x_{0}\right)^{3} .
\end{aligned}
$$

Remark 4.1 Note that we can match the observed level, slope and convexity of the implied volatility smile in the small-maturity limit, by the appropriate choice of $\sigma\left(x_{0}\right), \sigma^{\prime}\left(x_{0}\right)$ and $\sigma^{\prime \prime}\left(x_{0}\right)$, for a given exogenous choice of $f$ and $\alpha$.

\footnotetext{
${ }^{8}$ Using the triangle inequality and the Lipschitz property of $\sigma, f$ and $\alpha$, we can easily verify that the drift and the diffusion coefficients for this model satisfy the global Lipschitz and linear growth conditions in Theorem 2.9 in Karatzas\&Shreve[22], so a unique strong solution exists
} 
Remark 4.2 If we let $f(y) \equiv 1$ in (40), we recover the pure local volatility case and the well known formula $I^{\prime}\left(x_{0}\right)=$ $\sigma^{\prime}\left(x_{0}\right) / 2$, which has been proved heuristically in [7],[8]. For the pure uncorrelated stochastic volatility case $\sigma(x) \equiv 1$, $I\left(x_{1}\right)$ reduces to

$$
I\left(x_{1}\right)=f\left(y_{0}\right)+\frac{1}{6} \frac{f^{\prime}\left(y_{0}\right)^{2} \alpha\left(y_{0}\right)^{2}}{f\left(y_{0}\right)^{3}}\left(x_{1}-x_{0}\right)^{2}+O\left(x_{1}-x_{0}\right)^{4},
$$

and $I\left(x_{1}\right)$ is symmetric in the log-moneyness $x_{1}-x_{0}$.

Proof. We first consider the pure stochastic volatility case when $\sigma(x) \equiv 1$. Recall (5) and (6)

$$
\begin{aligned}
d\left(x_{1}\right) & =f\left(y_{1}^{*}\right) \int_{y_{0}}^{y_{1}^{*}} \frac{d y}{\alpha(y) \sqrt{f\left(y_{1}^{*}\right)^{2}-f(y)^{2}}}, \\
x_{1} & =\int_{y_{0}}^{y_{1}^{*}} \frac{f(y)^{2} d y}{\alpha(y) \sqrt{f\left(y_{1}^{*}\right)^{2}-f(y)^{2}}} .
\end{aligned}
$$

Set $g(y):=\int_{0}^{y} \frac{f(\xi)}{\alpha(\xi)} d \xi$ and $v:=g(y)$, so that $g^{\prime}(y)=\frac{f(y)}{\alpha(y)}, \frac{d v}{d y}(y)=g^{\prime}(y)$ and $f(y)=f \circ g^{-1}(v)=F(v)$. Then we can re-write the equations for $d\left(x_{1}\right)$ and $x_{1}$ as

$$
\begin{aligned}
d\left(x_{1}\right) & =F\left(v_{1}\right) \int_{v_{0}}^{v_{1}} \frac{d v}{F(v) \sqrt{F\left(v_{1}\right)^{2}-F(v)^{2}}}, \\
x_{1} & =\int_{v_{0}}^{v_{1}} \frac{F(v) d v}{\sqrt{F\left(v_{1}\right)^{2}-F(v)^{2}}},
\end{aligned}
$$

where $v_{0}=g\left(y_{0}\right)$ and $v_{1}=g\left(y_{1}^{*}\left(x_{1}\right)\right)$. Let $u=F(v)^{2}, u_{1}=F\left(v_{1}\right)^{2}, u_{0}=F\left(v_{0}\right)^{2}$. Expanding $f$ and $\alpha$ around $y=y_{0}$ we have

$$
\begin{aligned}
& f(y)=f\left(y_{0}\right)+f^{\prime}\left(y_{0}\right)\left(y-y_{0}\right)+\frac{1}{2} f^{\prime \prime}\left(y_{0}\right)\left(y-y_{0}\right)^{2}+O\left(y-y_{0}\right)^{3}, \\
& \alpha(y)=\alpha\left(y_{0}\right)+\alpha^{\prime}\left(y_{0}\right)\left(y-y_{0}\right)+O\left(y-y_{0}\right)^{2},
\end{aligned}
$$

so that

$$
F(v)=F_{0}+F_{1}\left(v-v_{0}\right)+\frac{1}{2} F_{2}\left(v-v_{0}\right)^{2}+O\left(v-v_{0}\right)^{3}
$$

where $F_{0}=f\left(y_{0}\right), F_{1}=\frac{f^{\prime}\left(y_{0}\right)}{g^{\prime}\left(y_{0}\right)}, F_{2}=\frac{f^{\prime \prime}\left(y_{0}\right)}{g^{\prime}\left(y_{0}\right)^{2}}-\frac{f^{\prime}\left(y_{0}\right) g^{\prime \prime}\left(y_{0}\right)}{g^{\prime}\left(y_{0}\right)^{3}}$. Then $F(v) d v=F(v) d u \frac{d v}{d u}=F(v) d u / \frac{d u}{d v}=d u / 2 F^{\prime}(v)$ and

$$
\begin{aligned}
& F^{\prime}(v)=F_{1}+F_{2}\left(v-v_{0}\right)+O\left(v-v_{0}\right)^{2}=F_{1}+\frac{F_{2}}{2 F_{1} \sqrt{u_{0}}}\left(u-u_{0}\right)+O\left(u-u_{0}\right)^{2}, \\
& \frac{1}{F^{\prime}(v)}=\frac{1}{F_{1}}-\frac{1}{2} \frac{F_{2}}{F_{1}^{3} \sqrt{u_{0}}}\left(u-u_{0}\right)+O\left(u-u_{0}\right)^{2} .
\end{aligned}
$$

Thus we have

$$
\begin{aligned}
x_{1} & =\frac{1}{2} \int_{u_{0}}^{u_{1}} \frac{\left(\frac{1}{F_{1}}-\frac{1}{2} \frac{F_{2}}{F_{1}^{3} \sqrt{u_{0}}}\left(u-u_{0}\right)+O\left(u-u_{0}\right)^{2}\right) d u}{\sqrt{u_{1}-u}}=\frac{1}{F_{1}} \sqrt{u_{1}-u_{0}}-\frac{F_{2}}{3 F_{1}^{3} \sqrt{u_{0}}}\left(u_{1}-u_{0}\right)^{\frac{3}{2}}+O\left(\left(u_{1}-u_{0}\right)^{5 / 2}\right) \\
& =\frac{\sqrt{2 F_{0}}}{\sqrt{F_{1}}} \sqrt{v_{1}-v_{0}}-\frac{1}{12} \sqrt{2} \frac{5 F_{0} F_{2}-3 F_{1}^{2}}{\sqrt{F_{0}} F_{1}^{3 / 2}}\left(v_{1}-v_{0}\right)^{\frac{3}{2}}+\left(v_{1}-v_{0}\right)^{\frac{5}{2}} .
\end{aligned}
$$

Inverting this series, we find that

$$
v_{1}=v_{0}+\frac{1}{2} \frac{F_{1}}{F_{0}} x_{1}^{2}+\frac{1}{24}\left(5 F_{0} F_{2}-3 F_{1}^{2}\right) F_{1} \frac{x_{1}^{4}}{F_{0}^{3}}+O\left(x_{1}^{6}\right) .
$$


Then we have

$$
\begin{aligned}
d\left(x_{1}\right) & =\frac{\sqrt{u_{1}}}{2} \int_{u_{0}}^{u_{1}} \frac{\left[\frac{1}{F_{1}}-\frac{1}{2} \frac{F_{2}}{F_{1}^{3} \sqrt{u_{0}}}\left(u-u_{0}\right)+O\left(u-u_{0}\right)^{2}\right] d u}{u \sqrt{u_{1}-u}} \\
& =\frac{1}{F_{1}} \tanh ^{-1}\left(\frac{\sqrt{u_{1}-u_{0}}}{\sqrt{u_{1}}}\right)-\frac{1}{2}\left[\sqrt{u_{1}-u_{0}} \sqrt{u_{1}}-u_{0} \tanh ^{-1}\left(\frac{\sqrt{u_{1}-u_{0}}}{\sqrt{u_{1}}}\right)\right] \frac{F_{2}}{F_{1}^{3} \sqrt{u_{0}}}+O\left(\left(u_{1}-u_{0}\right)^{2}\right) \\
& =\frac{\sqrt{u_{1}-u_{0}}}{\sqrt{u_{0}} F_{1}}+\left(-\frac{1}{6 u_{0}^{3 / 2} F_{1}}-\frac{1}{3} \frac{F_{2}}{u_{0} F_{1}^{3}}\right)\left(u_{1}-u_{0}\right)^{3 / 2}+O\left(\left(u_{1}-u_{0}\right)^{2}\right) \\
& =\frac{\sqrt{2}}{\sqrt{F_{1} F_{0}}} \sqrt{v_{1}-v_{0}}-\frac{1}{12} \frac{\sqrt{2}}{\left(F_{0} F_{1}\right)^{3 / 2}}\left(F_{1}^{2}+5 F_{0} F_{2}\right)\left(v_{1}-v_{0}\right)^{3 / 2}+O\left(\left(v_{1}-v_{0}\right)\right)^{5 / 2} \\
& =\frac{1}{F_{0}} x_{1}-\frac{1}{6} \frac{F_{1}^{2}}{F_{0}^{3}} x_{1}^{3}+O\left(x_{1}^{5}\right)=\frac{1}{f\left(y_{0}\right)} x_{1}-\frac{1}{6} \frac{f^{\prime}\left(y_{0}\right)^{2} \alpha\left(y_{0}\right)^{2}}{f\left(y_{0}\right)^{5}} x_{1}^{3}+O\left(x_{1}^{5}\right),
\end{aligned}
$$

and

$$
I(x)=x / d(x)=f\left(y_{0}\right)+\frac{1}{6} \frac{f^{\prime}\left(y_{0}\right)^{2}}{f\left(y_{0}\right)^{3}} \alpha\left(y_{0}\right)^{2} x^{2}+O\left(x^{4}\right) .
$$

For the general local-stochastic volatility case, we just combine (37) and (42), and the result follows.

\section{Numerical example: the Tanh model}

Consider the following choices for the drift and diffusion coefficients

$$
\begin{array}{ll}
f(y)=f_{0}+f_{1} \tanh \left(y-y_{c}\right), & \mu(y)=\kappa \tanh (\theta-y), \\
\alpha(y)=\alpha_{0}+\alpha_{1} \tanh \left(y-y_{c}\right), & \sigma(x)=\sigma_{0}+\sigma_{1} \tanh \left(x-x_{0}\right),
\end{array}
$$

with $f_{0}, f_{1}, \alpha_{0}, \alpha_{1}, \sigma_{0}>0, f_{0}>f_{1}, \alpha_{0}>\alpha_{1},\left|\sigma_{1}\right|<\sigma_{0}, \kappa>0$. Unlike the well known SABR and Heston models, this model satisfies all the conditions needed for Theorem 1.1, and we have enough flexibility to fit the level, slope and second derivative of the implied volatility smile at-the-money in the small-time limit. Note that $\mu(y)$ is mean-reverting, but $\kappa$ and $\theta$ will not affect the implied volatility in the small-time limit. Moreover, we can actually integrate (5) and (27) in closed form, although the answer is somewhat cumbersome so we omit the details. In Figures 1 and 2, we have plotted a family of geodesics and the implied volatility smile in the small-maturity limit for this model, using (9). 


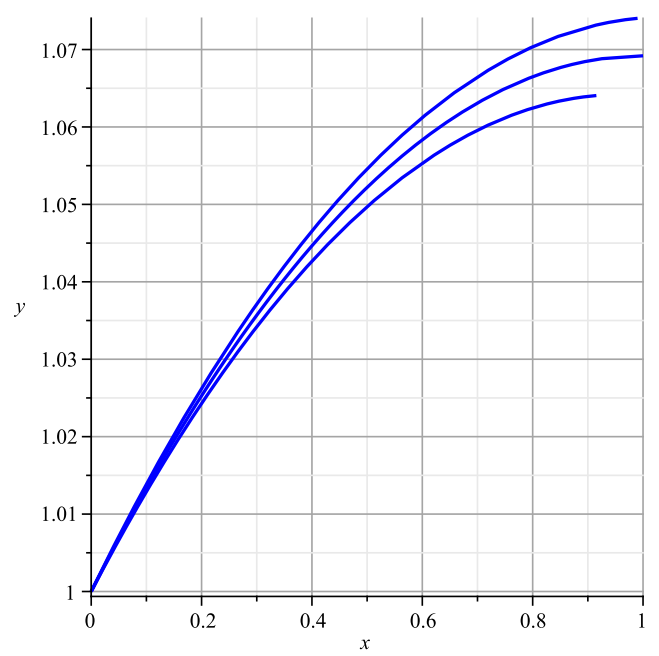

Figure 1: Here we have plotted a family of geodesics from $\left(x_{0}, y_{0}\right)=(0,1)$ to the line $\{x=1\}$ for the Tanh model, with $f_{0}=\alpha_{0}=1, f_{1}=\alpha_{1}=\frac{1}{2}, \sigma_{0}=.1, \sigma_{1}=-.001, y_{c}=0, x_{0}=0$. The middle curve is the shortest geodesic which comes in perpendicular to $\{x=1\}$.

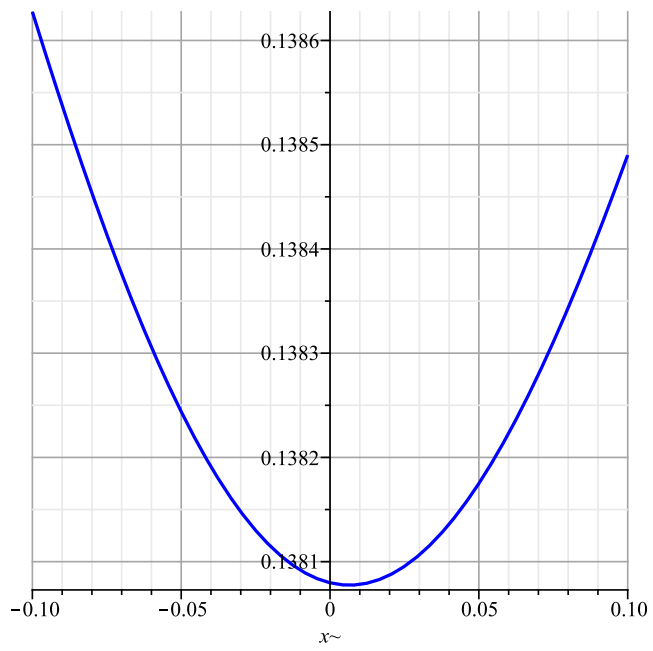

Figure 2: Here we have plotted the implied volatility (on the $y$-axis) as a function of the log-moneyness (on the x-axis) in the small-maturity limit for the Tanh model, with parameters given in the caption above, using the formula in (36).

\section{References}

[1] Berestycki, H., J. Busca and I. Florent, "Asymptotics and calibration of local volatility models", Quantitative Finance, 2, pgs 61-69, 2002.

[2] Berestycki H., J. Busca and I. Florent, "Computing the Implied Volatility in Stochastic Volatility models" Communications on Pure and Applied Mathematics, Vol LVII, pgs 1352-1373, 2004.

[3] Cox, J. (1975), "Notes on option pricing I: Constant elasticity of variance diffusions" working paper, Stanford university, reprinted in Journal of Portfolio Management, 22, 15-17, 1996.

[4] Carmo, M. do, "Riemmanian Geometry", Birkhäuser, 1992.

[5] Davydov, D. and V. Linetsky, "The Valuation and Hedging of Barrier and Lookback Options under the CEV Process," Management Science, 47, pgs 949-965, 2001.

[6] Dembo, A. and O.Zeitouni, "Large deviation techniques and applications", Jones and Bartlet publishers, Boston, 1993. 
[7] Derman, E., "The Local Volatility Surface: Unlocking the Information in Index Options Pricing", Financial Analysts Journal, July-Aug 1996, pgs 25-36, 1996.

[8] Durrleman, V., "From implied to spot volatilities", PhD dissertation, Princeton University, 2004.

[9] Durrleman, V., "From implied to spot volatilities", April 2005 version, revised version forthcoming in Finance and Stochastics.

[10] Feng, J., M.Forde and J.P.Fouque, "Short maturity asymptotics for a fast mean-reverting Heston stochastic volatility model", SIAM J. Finan. Math., Volume 1, pgs 126-141, 2010.

[11] Feng, J. and T.G.Kurtz, "Large Deviations for Stochastic Processes: Mathematical Surveys and Monographs", Vol 131, American Mathematical Society, 2006.

[12] Forde, M., "Small-time asymptotics for a general local-stochastic volatility model, using the heat kernel expansion", submitted, 2009.

[13] Forde, M. and A.Jacquier , "Small-time asymptotics for implied volatility under the Heston model", International Journal of Theoretical and Applied Finance, Vol 12, issue 6, pgs 861-876, 2009.

[14] Forde, M., A.Jacquier and R.Lee, "The small-time smile and term structure of implied volatility under the Heston model", 2010.

[15] Fouque, J.P, G. Papanicolaou, and R.K.Sircar, Derivatives in financial markets with stochastic volatility. Cambridge University Press, Cambridge, 2000.

[16] Freidlin, M.I. and A. Wentzell, "Random perturbations of dynamical systems", Second Edition, Springer-Verlag, New York, 1998.

[17] Gatheral, G., E.Hsu, P.Laurence, C.Ouyang and T-H.Wang, "Asymptotics of implied volatility in local volatility models" (2009), forthcoming in Mathematical Finance.

[18] Hagan, P., D. Kumar, A. S. Lesniewski and D. E.Woodward, "Managing Smile Risk", Wilmott Magazine, 2002.

[19] Henry-Labordère, P., "A General Asymptotic Implied Volatility for Stochastic Volatility Models", working paper, 2005.

[20] Henry-Labordère, P. , "Analysis, Geometry, and Modeling in Finance: Advanced Methods in Option Pricing", Chapman \& Hall, 2009.

[21] Islah, O., "Solving SABR in exact form and unifying it with LIBOR market model", working paper, 2009.

[22] Karatzas, I. and S.Shreve, "Brownian motion and Stochastic Calculus", Springer-Verlag, 1991.

[23] Laurence, P., "Asymptotics for local volatility and Sabr models", presentation at Leicester, 2009.

[24] Lewis, A., "Option valuation under stochastic volatility", Finance Press, 2000.

[25] Lewis, A., "Geometries and smile asymptotics for a class of Stochastic Volatility models", www.optioncity.net, 2007.

[26] Matsumoto, H. and Yor, M., "Exponential Functionals of Brownian motion I: Probability laws at fixed time, Probab. Surveys, 2, pgs 312-347, 2005.

[27] Minakshisundaram, S. and A. Pleijel, "Some properties of the eigenfunctions of the Laplace operator on Riemannian manifolds", Canadian J. Math. 1, pgs 242-256, 1949.

[28] Paulot, L., "Asymptotic Implied Volatility at the Second Order With Application to the SABR Model", working paper, 2009.

[29] Pham, H., "Large deviations in mathematical Finance", lecture notes, 2010, http://felix.proba.jussieu.fr/pageperso/pham/GD-finance.pdf.

[30] Roper, M. and M.Rutkowski, "A Note On The Behaviour Of The Black-Scholes Implied Volatility Close To Expiry", forthcoming in the International Journal of Theoretical and Applied Finance. 
[31] Robertson, S., "Sample Path Large Deviations and Optimal Importance Sampling for Stochastic Volatility Models", Stochastic Processes and their Applications, 120 (2010) 66-83.

[32] Varadhan, S.R.S., "On the behavior of the fundamental solution of the heat equation with variable coefficients", Comm. Pure Appl. Math., 20, pgs 431-455, 1967.

[33] Varadhan, S.R.S., "Diffusion processes in a small time interval", Comm. Pure Appl. Math., 20, pgs 659-685, 1967. 